\title{
Radio Interferometric Observations of NGC 2146
}

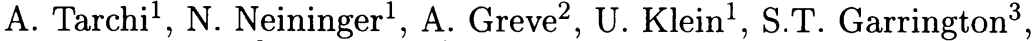 \\ T.W.B. Muxlow ${ }^{3}$, A. Pedlar ${ }^{3}$, B.E. Glendenning ${ }^{4}$ \\ 1 Radioastronomisches Institut der Universität Bonn (D); \\ ${ }^{2} \operatorname{IRAM}(F) ;{ }^{3} \mathrm{NRAL}(U K) ;{ }^{4} \mathrm{NRAO}$ (USA)
}

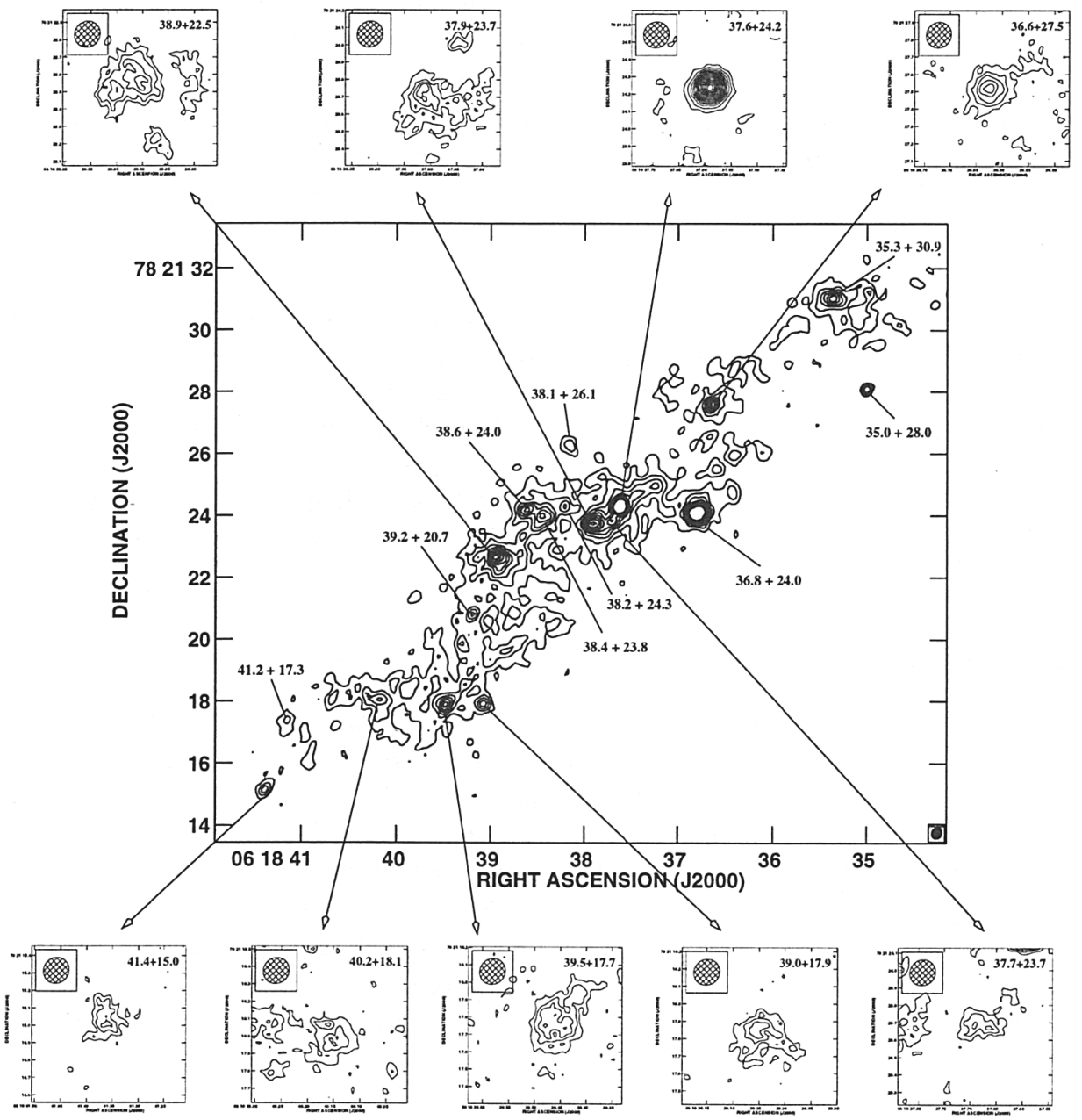

Figure 1. The $5 \mathrm{GHz}$ pure uniformly weighted A,B,C-array VLA map, with a beam (HPBW) of $0^{\prime \prime} 37 \times 0.32$. The smaller maps are sub-images of 9 sources from the MERLIN + VLA A-array uniformly weighted map (resolution 0 ! $15 \times 0$ '. 14). For these sources we have calculated spectral indices. 


\section{Summary}

At $5 \mathrm{GHz}$, about 20 point sources were detected earlier by Glendenning \& Kronberg (1986) in the central 800 pc of NGC 2146. Our observations with higher sensitivity and resolution made with MERLIN and the VLA confirms the detection of 18 sources, and resolves 7 of them (Fig.1). Additional 1.6-GHz MERLIN observations disclose 9 sources coincident in position with those detected at 5 $\mathrm{GHz}$, which allows us to derive their spectral indices $\alpha_{1.6}^{5},\left(\mathrm{~S}_{\nu} \sim \nu^{\alpha}\right)$. Only 3 sources have indices $(\alpha<0)$ consistent with synchrotron emission from supernova remnants or radio supernovae, while the others have very steep inverted spectra $(\alpha>0)$.

At $5 \mathrm{Ghz}$ one of the sources with negative spectral indices $(38.9+22.5)$ presents a partial shell structure with a diameter of $\sim 30 \mathrm{pc}$, though this is not clearly supported by the image of the source at $1.6 \mathrm{GHz}$ (Fig.2).

The nonthermal source $37.6+24.2$ has a position offset of $\sim 1$ ". 15 north-east of the dynamic center of NGC 2146 . It has a $5 \mathrm{GHz}$ flux density of $\sim 2 \mathrm{mJy}$ and it is not resolved even in a $5 \mathrm{GHz}$ MERLIN map with a resolution of 0 !'04, indicating that it is a very strong and compact "central" object (less than $3 \mathrm{pc}$ diameter). VLBI studies to understand the nature and morpholgy of this source are ongoing.

Two scenarios to explain the small number of SNRs/RSN found in NGC 2146 with respect to the strength of its starburst are presented and discussed in Tarchi et al. (2000).
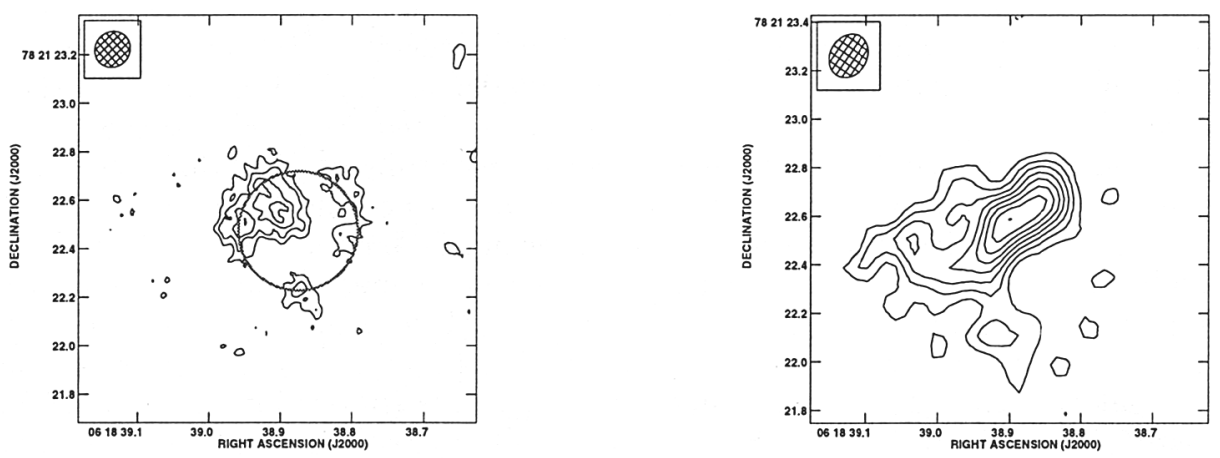

Figure 2. Left panel: source $38.9+22.5$ at $5 \mathrm{GHz}$; the contour interval is $0.05 \mathrm{mJy} /$ beam $(2 \sigma)$ with the first contour at $0.15 \mathrm{mJy} /$ beam $(4 \sigma)$. Right panel: source $38.9+22.5$ at $1.6 \mathrm{GHz}$; the contour interval is $0.05 \mathrm{mJy} /$ beam $(2 \sigma)$ with the first contour at $0.1 \mathrm{mJy} /$ beam $(3 \sigma)$.

Acknowledgments. We would like to acknoledge Dr. Peter Thomasson for his helpful support and Dr. Peter Biermann for useful discussions.

\section{References}

Glendenning B.E., Kronberg P.P., 1986, BAAS 18, 1006

Tarchi A., Neininger N., Greve A., et al., 2000, A\&A, 358, 95 